\title{
The influence of neuroticism and extraversion on the perceived burden of dementia caregivers: An exploratory study
}

\author{
Isabel González-Abraldes, José Carlos Millán-Calenti, Laura Lorenzo-López, Ana Maseda \\ Gerontology Research Group, Faculty of Health Sciences, University of A Coruña, Campus de Oza, E-15006, A Coruña, Spain
}

\begin{abstract}
Most studies reflect dementia caregivers usually sustain higher levels of burden compared to other caregivers. However, they do not consider variability within the caregiver, such as personality traits. The purpose of this study was to examine the influence of extraversion and neuroticism on dementia caregiver burden. A cross-sectional descriptive study was conducted with 33 caregivers looking after demented-patients. All caregivers had intense burden levels, and their personality, depression, anxiety and self-rated health were evaluated. Personality variables had important effects on the caregiver burden and consequences on their mental health. Neuroticism was significantly correlated with burden $(\mathrm{r}=0.6, \mathrm{p}<0.01)$, depression $(\mathrm{r}=0.68, \mathrm{p}<0.01)$ and both anxiety measures, state $(\mathrm{r}=0.46, \mathrm{p}$ $<0.01)$ and trait $(r=0.67, \mathrm{p}<0.01)$. Extraversion was significantly correlated with neuroticism $(\mathrm{r}=-0.42, \mathrm{p}<0.01)$ and burden $(r=-0.46, p<0.01)$. Finally, depression was significantly correlated with state $(r=0.63, p<0.01)$ and trait anxiety $(r=0.66, p<0.01)$. These results indicate the importance of considering the caregiver personality in the theoretical and empirical models of the caring process. It is necessary to adequately assess the caregiver personality, as those presenting high levels of neuroticism and low levels of extraversion are more vulnerable to experience negative caring effects.
\end{abstract}

Keywords: Anxiety, Burden, Caregiving, Dementia, Depression, Personality

\section{Introduction}

Different authors (Bedard, Pedlar, Martin, Malott, \& Stones, 2000; León-Salas, Olazarán, Muñiz, González-Salvador, \& Martínez-Martín, 2011) have established the relationships among behavior, cognitive and functional impairment, quality of life and health in people with dementia with the burden perceived by their caregivers (Fernández de Larrinoa Palacios et al., 2011). An increasing caregiver burden is known to decrease life satisfaction (Andrén \& Elmstahl, 2008). Diverse variables in reference to the care context and the caregiver as well as the care recipient show major associations with the burden: those associated to disease and the social situation of the dependent person, the greater frequency and intensity of care, and the low frequency in which the caregiver receives help from others (Garcés, Carretero, Ródenas, \& Sanjosé , 2009).

However, it is important to highlight that, in general, those studies are focused on the burden of immediate factors such as the impact of the cognitive deficits, help with everyday functioning and behavioral and psychological factors. Most of them do not take into consideration variability within the caregivers, such as personality traits (Campbell et al., 2008; Löckenhoff, Duberstein, Friedman, \& Costa, 2011).

Personality traits are defined as "dimensions of individual differences in tendencies to show consistent patterns of thoughts, feelings and actions" (McCrae \& Costa, 1990). Some of these traits, such as neuroticism and extraversion, have been related to caregiver burden (Koerner, Kenyon, \& Shirai, 2009). Furthermore, few existing studies within the personality field have focused on the extraversion variable in its relationship to caregiving (Melo, Maroco, \& Mendonça, 2011).

Neuroticism or negative affectivity is a personality trait that reflects people's tendency to experience negative emotional states. It is one of the five main personality dimensions, and it is conceptualized as the underlying tendency to experience emotional distress. It is a stable trait that reflects the tendency to focus on the negative aspects of the situation (Carson, 1989). People with high levels of neuroticism have been labeled as a person who is a "worrier, nervous, emotional, with low self-esteem, inadequate and hypochondriacal" (Costa \& McCrae, 1985). Neuroticism has also been described as a psychological distress construct (Costa \& McCrae, 1985; Lincoln, 2008). Consequently, high neuroticism scores have 
been consistently related to poor mental health (Lincoln, 2008). Highly neurotic individuals have been shown to appraise situations as more stressful or threatening and have negative perceptions of physical problems (Jang, Mortimer, Haley, \& Graves, 2002). Neuroticism is a risk factor for increased caregiver distress over time, and as it is easily identified, it should be targeted for intervention (Jang, Clay, Roth, Haley, \& Mittelman, 2004).

In contrast, extraversion is a global indicator of a person's tendency to look for social interactions and activities. According to Costa and McCrae (1985), high scores on the extraversion scale are associated with sociable, active, talkative, person-oriented, optimistic, fun-loving, affectionate people who like to enjoy themselves, while low scores are associated with reserved, sober, unexuberant, aloof, task-oriented and retiring quiet people. Extraversion or the wish for high levels of social interaction is related to "positive affectivity". Extroverted caregivers feel more positive toward their efforts in caregiving, find more pleasing aspects in the task and are more satisfied with the social support they get from family and friends. In addition, they are more likely to carry out leisure activities with other people that help to reduce the feeling of burden and safeguard their health (Hirano et al., 2011). However, caregivers who score low in extroversion may have fewer buffers and resources when faced with daily caregiving stressors and may be less able to derive any benefits or rewards from the caregiving experience (Koerner et al., 2009). Few existing studies within the personality field have focused on the extraversion variable in its relationship to caregiving.

Neuroticism-emotional adjustment and extraversion-introversion are basic constructs in all theories of personality and have been strongly related to affectivity and well-being (Hotard, McFatter, McWhirter, \& Stegall, 1989). Personality is one such personal resource in the caregiver stress process, and accumulating evidence suggests that neuroticism may be especially predictive of adverse caregiver outcomes (Gallant \& Connell, 2003)

As personality variables have been consistently associated with affective states, we should take such variables into account when attempting to understand the consequences of caregiving. Personality does help to account for the experience of caregiving benefits/gains (Koerner et al., 2009).

Another aspect that needs to be considered is the mental health of the caregiver. Depression is a common problem among dementia-caregiving families (Joling, Smit, et al., 2012) and higher levels of burnout were found to correspond to higher levels of depressive symptoms (Takai et al., 2009). It normally lasts for long periods of time, and its influence on caregivers has been widely studied. In contrast, studies of anxiety, together with worry and feelings of fear, and psychological symptoms such as muscular tension and somatic symptoms among dementia-caregiving families have been somewhat neglected (Cooper, Balamurali, \& Livingston, 2007).

Despite the fact that prevalence studies report anxiety rates from $3.7 \%$ to $76.5 \%$, when using more reliable assessment methods in longitudinal studies, approximately $25 \%$ of caregivers report clinically significant anxiety experiences (Joling, van Marwijk, et al., 2012; Mahoney, Regan, Katona, \& Livingston, 2005).

Given the fact that few studies have demonstrated the importance of personality factors, neuroticism and extraversion, among caregivers with severe burden in the caring experience of the patients with dementia, the aim of our study was 2-fold: (1) to analyze the influence that 2 personality traits (neuroticism and extraversion) have on dementia caregiver self-rated burden and (2) to examine the possible relationship between caregiver personality and psychological morbidity (depression and anxiety).

\section{Materials and methods}

\subsection{Selection and description of participants}

Participants were recruited from the Association of Relatives of Sufferers of Alzheimer's or Other Dementias and an Elderly Care Center of the City of A Coruña, Spain. Questionnaire packets were sent to 109 caregivers, and $41(36.7 \%)$ returned completed questionnaires, of whom 8 did not meet the inclusion criteria for severe burden level. Overall, 33 primary caregivers ranging in age from 35 to 82 years $(\mathrm{M}=$ $57.5, \mathrm{SD}=11.3$ years) took part in this study.

Each caregiver completed the self-administered assessment according to the recommendations given by a clinical psychologist with experience working with patients diagnosed with dementia and their informal caregivers.

The inclusion criteria were as follows: being the primary caregiver (persons were designated as the primary caregiver if they were principally responsible for providing or coordinating the resources required by the person with dementia, such as housekeeping, financial help and shopping) of a person with dementia for at least the previous 6 months, irrespective of whether the caregiver lived with the person or not; providing the major source of unpaid elder care; the caregiving presented a severe burden 
(score on the Zarit Burden Scale higher than 55 according to the reference values proposed by Martín et al., 1996); and giving informed consent.

Caregivers were excluded if they did not possess the necessary skills to be assessed. Exclusion criteria for caregivers included cognitive impairment, illiteracy or severely impaired vision and hearing.

\subsection{Procedure}

The study protocol was approved by the Ethics Committee at the University of A Coruña (Spain). Before the data collection, all participants were informed about the study and signed the corresponding Informed Consent.

\subsection{Variables and instruments}

\subsubsection{Patient's cognitive and functional status}

Patient's dementia stages were assessed by the Clinical Dementia Rating (CDR) (Hughes, Berg, Danziger, Coben, \& Martin, 1982; Morris, 1993). The CDR is a clinical staging instrument for dementia. It characterizes 6 domains of cognitive and functional performance: Memory, Orientation, Judgment and Problem Solving, Community Affairs, Home and Hobbies, and Personal Care. The necessary information to make each rating is obtained through a semi-structured interview of the patient and a reliable informant (e.g., a family member). In addition to ratings on a 5-point scale for each domain (except Personal Care, which is rated on a 4-point scale) an overall CDR score is derived by standard algorithm. This score is useful for globally staging the level of impairment: $0=$ no impairment, $0.5,1,2$, and 3 indicate very mild, mild, moderate and severe dementia.

Regarding care recipient's characteristics $(\mathrm{n}=33), 9.1 \%$ suffered from mild dementia, $51.5 \%$ moderate dementia and $39.4 \%$ severe dementia according to CDR.

Caregivers completed a demographic questionnaire that recorded information on caregiver gender, age, marital status, educational level, occupation, relationship to the patient, duration of caring, date of diagnosis (as an indication of illness duration) and level of health and social support.

\subsubsection{Caregiver burden}

The level of burden experienced by the informal caregiver was measured by the Zarit Burden Interview (ZBI) (Zarit, Reever, \& Bach-Peterson, 1980), adapted and validated for the Spanish language by Martín et al. (1996). The ZBI was used to assess how much the caring affected the informal caregivers' health, social life, emotional welfare and other factors. This instrument consists of 22 items measured using a Likert scale (1, never, to 5, almost always). Three cut-off scores were established: no burden (22-46), slight burden (47-55), and severe burden (56-110).

\subsubsection{Personality traits (neuroticism and extraversion)}

Personality was assessed by the NEO Five-Factor Inventory (NEO-FFI) (Costa \& McCrae, 2008). This is a 60 -item questionnaire comprising 12 items representing each of the facets encompassed by the 5 -factor model of personality (neuroticism, extraversion, openness to experience, agreeableness and conscientiousness).

Nevertheless, in this study, only two 12-item scales, neuroticism and extraversion, were assessed. These personality dimensions were selected due to their important influence on caregiver burden. Previous studies indicate that caregivers that present higher levels of neuroticism (Campbell et al., 2008) and lower levels of extroversion (Koerner et al., 2009) report a higher level of burden. The respondents were required to endorse each item on a 5-point scale ranging from 1 (strongly disagree) to 5 (strongly agree), as it applied to them.

The obtained values can be specified at several levels to establish the degree of neuroticism: very low, a score between 0 and 5; low, a score between 6 and 11; medium, a score between 12 and 17; high, a score between 18 and 26; and very high, a score between 27 and 48. The extraversion score was defined as follows: very low, a score between 0 and 22; low, a score between 23 and 29; medium, a score between 30 and 35; high, a score between 36 and 41; and very high, a score between 42 and 48.

\subsubsection{Mental health (depression and anxiety)}

To assess the symptoms of depression, the caregivers were also administered the Spanish version (Sanz \& Vázquez, 1998) of the Beck Depression Inventory (BDI) (Beck, Ward, Mendelson, Mock, \& 
Erbaugh, 1961). The BDI is a widely used 21-item self-report instrument. Each item is rated on a 4-point scale that represents the presence and severity of a given symptom, as rated from 0 to 3 . The total scores can range from 0 to 63 . The scores were used to distinguish depression levels as follows: none or minimal depression (9 or less), mild depression (10-18), moderate depression (19-29) and severe depression (30 or more) (Beck, Steer, \& Garbin, 1988).

Anxiety was assessed using the Spanish version of the State-Trait Anxiety Inventory (STAI) (Spielberger, Gorsuch, \& Lushene, 1970), validated and adapted by TEA editions (TEA Ediciones, 2008). The STAI is one of the most frequently used measures of anxiety, being a rapid self-report scale for assessing state and trait anxiety. The instrument asks individuals to rate the symptoms of anxiety on a 4point scale from 1 to 4 . The Spanish adaptation of this scale contains 40 items rated on a 4-point scale (03 ) and is designed to measure the levels of state anxiety (20 items, situational and transient anxiety) and trait anxiety (20 items, dispositional and stable anxiety). The total scores vary from 0 to 60 , rather than the 20 to 80 in the original version. Decatypes have been used to assess anxiety levels following the STAI manual instructions.

\subsubsection{Self-rated health}

This variable was measured with the question "In general, how would you rate your health?" Participants could give 5 possible responses: very good, good, fair, poor and very poor.

\subsection{Statistical analysis}

SPSS for Windows version 16.0.1 was used to perform Pearson's correlation tests between all the variables assessed: burden, neuroticism, extraversion, depression, anxiety, and self-rated health. A multiple linear regression analysis was performed to determine the influence of the independent variables to predict burden. The perceived burden was used as the dependent variable, and the others variables were introduced into the model as covariables. To control for possible multicollinearity, we examined all correlations of the independent variables considering that a condition index greater than 30 suggests a serious problem with collinearity (Liu, Kuang, Gong, \& Hou, 2003). Statistical significance was set at p < 0.05 .

\section{Results}

The sociodemographic characteristics of the caregivers included in the analysis are listed in Table 1.

After applying the inclusion/exclusion criteria, 33 caregivers demonstrated severe burden according to the ZBI $(M=69.9, \mathrm{SD}=7.8)$. Regarding caregiver personality, high levels of neuroticism $(\mathrm{M}=22.6, \mathrm{SD}$ $=8.3)$ and low extraversion $(\mathrm{M}=26.0, \mathrm{SD}=7.9)$ were displayed. Most of the caregivers presented depressive symptoms according to the BDI. The mean score was $13.8(\mathrm{SD}=7.6)$, indicating a level of mild depression. The caregivers had higher levels of state anxiety $(\mathrm{M}=6.6 ; \mathrm{SD}=1.5)$ than of trait anxiety $(\mathrm{M}=5.8, \mathrm{SD}=1.9)$. The mean anxiety score of the sample was $5.5(\mathrm{SD}=2.0)$.

In order to examine strength of relationships between burden, personality factors, caregiver mental health and self-rated health, correlations between these variables were performed.

As shown in Table 2, neuroticism was significantly correlated with burden and with the variables of mental health, depression and both anxiety measures (state and trait). Those caregivers with high scores in neuroticism considered themselves to be more burdened and presented more depressive symptoms and anxiety.

Extraversion was significantly correlated with neuroticism and with burden. Caregivers with low scores on the extraversion scale had high scores in neuroticism and higher self-reported burden.

As expected, depression was significantly correlated with state anxiety and trait anxiety. That is, those caregivers with high scores in depression also presented anxiety symptoms.

The caregiver burden was significantly correlated with neuroticism, extraversion, depression and trait anxiety. Caregivers with severe burden presented high scores in neuroticism and low extraversion scores. Their self-rated burden is associated with their mental health.

Finally, mental health (assessed with the item: How do you perceive your health state?) was significantly correlated with burden, neuroticism, extraversion, and trait anxiety. 
Table 1

Sociodemographic characteristics of the caregivers included in the analysis $(n=33)$.

\begin{tabular}{lll}
\hline & $\begin{array}{l}\text { Mean/ } \\
\text { frequency }\end{array}$ & $\begin{array}{l}\text { SD (range)/ } \\
\text { percentage }\end{array}$ \\
\hline Age (years) & 57.6 & $11.3(35-82)$ \\
Sex & 28 & 84.8 \\
$\quad$ Female & 5 & 15.2 \\
Male & & \\
Family tie & 21 & 63.6 \\
Children & 9 & 27.3 \\
Spouse & 3 & 9.1 \\
Others (nieces) & & 75.8 \\
Marital status & 25 & 18.2 \\
Married & 6 & 6.0 \\
Single & 2 & 18.2 \\
Divorced & & 3.0 \\
Educational level & 6 & 48.5 \\
0-4 years & 1 & 30.3 \\
5-8 years & 16 & \\
9-12 years or high school diploma & 10 & \\
College experience or higher degree & & \\
\end{tabular}

Table 2

Correlation matrix of burden, personality, mental health and self-rated health variables.

\begin{tabular}{|c|c|c|c|c|c|c|c|}
\hline & 1 & 2 & 3 & 4 & 5 & 6 & 7 \\
\hline 2. Neuroticism & $0.60 * *$ & - & & & & & \\
\hline 3. Extraversion & $-0.46^{* *}$ & $-0.42 * *$ & - & & & & \\
\hline 4. Depression & $0.53 * *$ & $0.68 * *$ & -0.13 & - & & & \\
\hline 7. Self-rated health & $-0.51 * *$ & $-0.46^{* *}$ & $0.35^{*}$ & -0.32 & -0.28 & $-0.37 *$ & - \\
\hline
\end{tabular}

* Correlation significant at the 0.05 level.

** Correlation significant at the 0.01 level.

The results of the multiple regression models using the caregiver burden as the dependent variable and the rest of variables (neuroticism, extraversion, depression, state and trait anxiety) as independent variables, are listed in Table 3. The independent effects of the predictor variables on caregiver burden were not statically significant. Nevertheless, the coefficient of determination (R2) and the significant F test $(\mathrm{p}<0.001)$ of the combined effect of all these predictors simultaneously thrown in the model, would explain $38 \%$ of the caregiver burden (adjusted R2). No harmful multicollinearity (correlation between one of the independent variables and some linear combination of the remaining ones) was detected.

Table 3

Multivariate regression model for variables predicting subjective caregiver burden.

\begin{tabular}{llll}
\hline Dependent variables & \multicolumn{2}{l}{ Subjective burden } & \\
\cline { 2 - 4 } Independent variables & Beta & $\mathrm{T}$ & $\mathrm{p}$ value \\
\hline & & & \\
Neuroticism & 0.20 & 0.9 & 0.37 \\
Extraversion & -0.29 & -1.8 & 0.08 \\
Depression & 0.38 & 1.6 & 0.12 \\
State anxiety & -1.01 & -1.0 & 0.35 \\
Trait anxiety & 0.64 & 0.7 & 0.50 \\
& & & \\
\hline
\end{tabular}

$\mathrm{R}^{2}=0.48 ;$ adjusted $\mathrm{R}^{2}=0.38 ; \mathrm{F} 2,30=4.94(0.002)$. 


\section{Discussion}

Our results support the idea that the personality variables assessed (neuroticism and extraversion) strongly affect self-rated caregiver burden. This is consistent with previous studies that relate neuroticism with a higher self-rated stress and burden (Campbell et al., 2008; Jang et al., 2004; Oken, Fonareva, \& Wahbeh, 2011). The burden in caregivers of elderly with dementia is due to restrictions on their personal time and to the sense of failure regarding their hopes and expectations (Luchetti et al., 2009).

There are very few studies that relate the personality variable extraversion with burden. In our study, we found that caregivers with high levels of neuroticism and low levels of extraversion experienced higher burden and had worse mental health. Melo et al. (2011) found that high scores in extraversion were associated with low levels of neuroticism and better health. Moreover, mental health and burden were significantly associated with caregivers' health problems simultaneously (Chang, Chiou, \& Chen, 2010).

Other authors assessed similar variables, such as dispositional optimism, finding that individuals with worse mental health presented higher scores in neuroticism and lower scores in optimism (Sharpe, Martin, \& Roth, 2011).

The personality variables are related to caregiver mental health. Mental health is an indication of adaptation to life experiences. It is sometimes argued that personality has an effect on mental health because it channels the experiences one is likely to encounter. For example, highly neurotic people may seek out negative life experiences that lead naturally to depression or poorer well-being (Hooker, Monahan, Bowman, Frazier, \& Shifren, 1998). Therefore, such as in our study, highly neurotic caregivers experience a greater amount of stress and depressive symptoms compared with less neurotic caregivers (Hooker et al., 1998; Jang et al., 2004). The results of this study are consistent with those of Gallant and Connell (2003) who stated that neuroticism had a significant direct effect on depressive symptoms. Highly neurotic caregivers were more likely to appraise the caregiving situations as more stressful.

In addition, previous studies with caregivers found that high optimism, high extraversion and low neuroticism are related to better coping with elderly caregiving (Marziali \& Garcia, 2011). In this context, increased examination of characteristics could be modified by psycho-educational programs to informal caregivers is essential to provide them with skills and coping strategies (Garcés, Carretero, Ródenas, \& Vivancos, 2010). Moreover, support programs have a positive impact on the family carers since they feel supported (Millán-Calenti et al., 2000).

Lastly and similar to other authors (Cooper et al., 2007), we found evidence relating caregiver selfrated burden to anxiety and self-rated burden, a factor associated with an increased risk of suffering depressive symptoms.

Although the aim of this study was not to determine causality since the results are based upon-cross sectional data, our main results suggested that personality traits had important effects on the perceived caregiver burden and relevant consequences on their mental health. However, we cannot completely rule out the possibility that long-term and intensive caring of demented patients has, at the same time, some effect on caregiver's personality traits. Another likely possibility is reciprocal causation. In this regard, vulnerable personality trait levels may make caregivers more distressed when faced with such obligations as taking care for demented patients but the distress, in turn, may make their personality trait levels even more vulnerable. All these aspects should be taking into account in future studies.

Finally, though the sample size was small, there was maximum meticulousness used in developing the inclusion criteria for determining the study sample. These selective criteria required the participants to be caring for someone with a previous dementia diagnosis, as well as a high caregiving burden based on the instrument used. These necessary conditions reduced the sample size. Despite this limitation, there is still consistent evidence that proves the influence of caregiver personality on burden perception and it could be a starting point for a thorough analysis of the influence of extraversion and neuroticism on dementia caregiver burden in further researches.

\section{Conclusion}

In conclusion, these results indicate that caregiver personality should be included in the models of the caregiving coping process. Assessments of caregiver personality should be carried out in high perceived burden caregivers, as highly neurotic caregivers and those with low extraversion are more vulnerable to negative caregiving effects and should be targeted by early interventions that provide coping strategies training. 


\section{Conflict of interest statement}

None.

\section{Acknowledgments}

The authors sincerely thank the Association of Relatives of Sufferers of Alzheimer's or Other Dementias (AFACO) and the Elderly Care Centers of the City of A Coruña (Spain) for providing access to the caregivers. The authors also thank the caregivers participating in this study for helping to increase the knowledge of care tasks.

\section{References}

Andrén, S., \& Elmstahl, S. (2008). The relationship between caregiver burden, caregivers' perceived health and their sense of coherence in caring for elders with dementia. Journal of Clinical Nursing, 17(6), 790-799.

Beck, A. T., Steer, R. A., \& Garbin, M. G. (1988). Psychometric properties of the Beck Depression Inventory. Twenty-five years of evaluation. Clinical Psychology Review, 8, 77-100.

Beck, A. T., Ward, C. H., Mendelson, M., Mock, J., \& Erbaugh, J. (1961). An inventory for measuring depression. Archives of General Psychiatry, 4, 561-571.

Bedard, M., Pedlar, D., Martin, N. J., Malott, O., \& Stones, M. J. (2000). Burden in caregivers of cognitively impaired older adults living in the community: Methodological issues and determinants. International Psychogeriatrics, 12, $307-332$.

Campbell, P., Wright, J., Oyebode, J., Job, D., Crome, P., Bentham, P., et al. (2008). Determinants of burden in those who care for someone with dementia. International Journal of Geriatric Psychiatry, 23, 1078-1085.

Carson, R. C. (1989). Personality. Annual Review of Psychology, 40, 227-248.

Chang, H. Y., Chiou, C. J., \& Chen, N. S. (2010). Impact of mental health and caregiver burden on family caregivers' physical health. Archives of Gerontology and Geriatrics, 50(3), 267-271.

Cooper, C., Balamurali, T. B., \& Livingston, G. (2007). A systematic review of the prevalence and covariates of anxiety in caregivers of people with dementia. International Psychogeriatrics, 19, 175-195.

Costa, P. T., \& McCrae, R. R. (2008). NEO PI-R, revised NEO Personality Inventory and NEO Five Factor Inventory (NEO-FFI). Madrid: TEA. (in Spanish).

Costa, P. T., \& McCrae, R. R. (1985). The NEO Personality Inventory Manual. Odessa, FL: Psychological Assessment Resources.

Fernández de Larrinoa Palacios, P., Martínez Rodríguez, S., Ortiz Marqué s, N., Carrasco Zabaleta, M., Solabarrieta Eizaguirre, J., \& Gómez Marroquín, I. (2011). Family caregiver's self-perceived health status and its relation to burden. Psicothema, 23(3), 388-393.

Gallant, M. P., \& Connell, C. M. (2003). Neuroticism and depressive symptoms among spouse caregivers: Do health behaviors mediate this relationship? Psychology and Aging, 18, 587-592.

Garcés, J., Carretero, S., Ródenas, F., \& Sanjosé, V. (2009). Variables related to the informal caregivers' burden of dependent senior citizens in Spain. Archives of Gerontology and Geriatrics, 48(3), 372-379.

Garcés, J., Carretero, S., Ródenas, F., \& Vivancos, M. (2010). The care of the informal caregiver's burden by the Spanish public system of social welfare: A review. Archives of Gerontology and Geriatrics, 50(3), 250-253.

Hirano, A., Suzuki, Y., Kuzuya, M., Onishi, J., Ban, N., \& Umegaki, H. (2011). Influence of regular exercise on subjective sense of burden and physical symptoms in community-dwelling caregivers of dementia patients: A randomized controlled trial. Archives of Gerontology and Geriatrics, 53(2), e158-e163.

Hooker, K., Monahan, D., Bowman, S., Frazier, L., \& Shifren, K. (1998). Personality counts for a lot: Predictors of mental and physical health of spouses caregivers in two disease groups. Journal of Gerontology. Series B, Psychological Sciences and Social Sciences, 53B, P73-P85.

Hotard, S. R., McFatter, R. M., McWhirter, R. M., \& Stegall, M. E. (1989). Interactive effects of extraversion, neuroticism, and social relationships on subjective wellbeing. Journal of Personality and Social Psychology, 57, 321-331.

Hughes, C. P., Berg, L., Danziger, W. L., Coben, L. A., \& Martin, R. L. (1982). A new clinical scale for the staging of dementia. British Journal of Psychiatry, 140, 566-572.

Jang, Y., Clay, O. J., Roth, L., Haley, W. E., \& Mittelman, M. S. (2004). Neuroticism and longitudinal change in caregiver depression: Impact of a spouse-caregiver intervention program. Gerontologist, 44, 311-317.

Jang, Y., Mortimer, J. A., Haley, W. E., \& Graves, A. B. (2002). The role of neuroticism in the association between performancebased and selfreported measures of mobility. Journal of Aging and Health, 14, 495-508.

Joling, K. J., Smit, F., van Marwijk, H. W., van der Horst, H. E., Scheltens, P., Schulz, R., et al. (2012). Identifying target groups for the prevention of depression among caregivers of dementia patients. International Psychogeriatrics, 24(2), $298-306$.

Joling, K. J., van Marwijk, H. W. J., Smit, F., van der Horst, H. E., Scheltens, P., van de Ven, P. M., et al. (2012). Does a family meetings intervention prevent depression and anxiety in family caregivers of dementia patients? A randomized trial. PLoS ONE, 7(1), e30936.

Koerner, S. S., Kenyon, D. B., \& Shirai, Y. (2009). Caregiving for elder relatives: Which caregivers experience personal benefits/gains? Archives of Gerontology and Geriatrics, 48, 238-245.

León-Salas, B., Olazarán, J., Muñ iz, R., González-Salvador, M. T., \& Martínez-Martín, P. (2011). Caregivers' estimation of patients' quality of life (QoL) in Alzheimer's disease (AD): An approach using the ADRQL. Archives of Gerontology and Geriatrics, 53(1), 13-18.

Lincoln, K. D. (2008). Personality, negative interactions, and mental health. Social Service Review, 82(2), $223-252$.

Liu, R. X., Kuang, J., Gong, Q., \& Hou, X. L. (2003). Principal component regression analysis with SPSS. Computer Methods and Programs in Biomedicine, 71, 141-147.

Löckenhoff, C. E., Duberstein, P. R., Friedman, B., \& Costa, P. T. (2011). Five-factor personality traits and subjective health among caregivers: The role of caregiver strain and self-efficacy. Psychology and Aging, 26(3), 592-604. 
Luchetti, L., Uhunmwangho, E., Dordoni, G., Lorido, A., Barbieri, S., Bolognesi, A. G., et al. (2009). The subjective feeling of burden in caregivers of elderly with dementia: How to intervene? Archives of Gerontology and Geriatrics, 49(Suppl 1), 153161.

Mahoney, R., Regan, C., Katona, C., \& Livingston, G. (2005). Anxiety and depression in family caregivers of people with Alzheimer's disease: The LASER-AD study. American Journal of Geriatric Psychiatry, 13, 795-801.

Martín, M., Salvado, I., Nadal, S., Miji, L. C., Rico, J. M., Lanz, P., et al. (1996). Adaptation to our environment of the Zarit Caregiver Burden interview. Revista de Gerontología, 6, 338-346, (in Spanish).

Marziali, E., \& García, L. J. (2011). Dementia caregivers' responses to 2 Internet-based intervention programs. American Journal of Alzheimer's Disease and Other Dementias, 26(1), 36-43.

McCrae, R. R., \& Costa, P. T. (1990). Personality in adulthood. New York: Guilford Press.

Melo, G., Maroco, J., \& Mendonça, A. (2011). Influence of personality on caregiver's burden, depression and distress related to the BPSD. International Journal of Geriatric Psychiatry, 26, 1275-1282.

Millán-Calenti, J. C., Gandoy-Crego, M., Antelo-Martelo, M., López-Martinez, M., Riveiro-López, M. P., \& Mayán-Santos, J. M. (2000). Helping the family carers of Alzheimer's patients: From theory...to practice. A preliminary study. Archives of Gerontology and Geriatrics, 30(2), 131-138.

Morris, J. C. (1993). The Clinical Dementia Rating (CDR): Current version and scoring rules. Neurology, 43, 2412-2414.

Oken, B. S., Fonareva, I., \& Wahbeh, H. (2011). Stress-related cognitive dysfunction in dementia caregivers. Journal of Geriatric Psychiatry and Neurology, 24(4), 191-198.

Sanz, J., \& Vázquez, C. (1998). Reliability, validity, and normative data of the Beck Depression Inventory. Psicothema, 10, 303318 , (in Spanish).

Sharpe, J. P., Martin, N. R., \& Roth, K. A. (2011). Optimism and the big five factors of personality: Beyond neuroticism and extraversion. Personality and Individual Differences, 51, 946-951.

Spielberger, C. D., Gorsuch, R. L., \& Lushene, R. E. (1970). STAI, Manual for the State-Trait Anxiety Inventory (self-evaluation questionnaire). Palo Alto, CA: Consulting Psychologist.

Takai, M., Takahashi, M., Iwamitsu, Y., Ando, N., Okazaki, S., Nakajima, K., et al. (2009). The experience of burnout among home caregivers of patients with dementia: Relations to depression and quality of life. Archives of Gerontology and Geriatrics, 49(1), e1-e5.

TEA Ediciones. (2008). STAI, Manual for the State-Trait Anxiety Inventory (7th ed.). Madrid: TEA. (in Spanish).

Zarit, S. H., Reever, K. E., \& Bach-Peterson, J. (1980). Relatives of the impaired elderly: Correlates of feelings of burden. Gerontologist, 20, 649-655. 